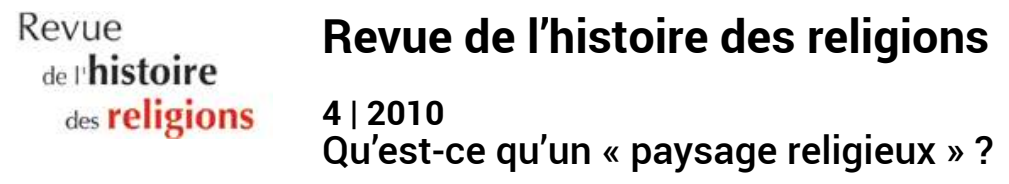

\title{
Le paysage religieux de Maktar à l'époque préromaine : l'aire sacrée de Baal Hammon
}

The Religious Landscape of Maktar in the Pre-Roman Period: The Sacred Space

of Baal Hamon

Lamia Ben Abid

\section{CpenEdition}

\section{Journals}

Édition électronique

URL : http://journals.openedition.org/rhr/7688

DOI : $10.4000 /$ rhr.7688

ISSN : 2105-2573

Éditeur

Armand Colin

Édition imprimée

Date de publication : 1 décembre 2010

Pagination : 683-701

ISBN : 978-2200-92658-8

ISSN : 0035-1423

\section{Référence électronique}

Lamia Ben Abid, « Le paysage religieux de Maktar à l'époque préromaine : l'aire sacrée de Baal

Hammon », Revue de l'histoire des religions [En ligne], 4 | 2010, mis en ligne le 01 décembre 2013

consulté le 30 avril 2019. URL : http://journals.openedition.org/rhr/7688 ; DOI : 10.4000/rhr.7688

Tous droits réservés 


\section{Le paysage religieux de Maktar à l'époque préromaine : l'aire sacrée de Baal Hammon}

Le site antique de Maktar en Tunisie renferme plusieurs vestiges se rapportant à des lieux de culte préromains. Ces ruines ont été dégagées à la fin $d u X X X^{e}$ siècle et au début $d u X X^{e}$. Cependant les résultats publiés de ces travaux ne permettent pas de reconstituer le paysage religieux de cette cité avant son annexion par les Romains. À travers une étude détaillée des circonstances qui ont entouré la découverte des ex-voto relatifs au culte de Baal Hammon et des indications historiques que peut fournir l'étude iconologique de ces monuments, ce texte illustre les problèmes que posent l'interprétation des sources archéologiques et la difficulté, en l'absence d'indications dans les sources classiques, de constituer une topographie religieuse pour cette ville notamment à l'époque préromaine.

\section{The Religious Landscape of Maktar in the Pre-Roman Period : The Sacred Space of Baal Hamon}

The ancient site of Maktar in Tunisia contains several archaeological remnants of pre-Roman worship sites. The ruins were exposed at the end of the $19^{\text {th }}$ and beginning of the $20^{\text {th }}$ century. However, the published results of these efforts do not make it possible to reconstitute the religious landscape of this city before its annexation by the Romans. Through a detailed study of the circumstances surrounding the discovery of ex-votos related to the cult of Baal Hamon as well as some historical indications provided by the iconological study of these monuments, this text illustrates the problems posed by the interpretation of archaeological sources. Due to the lack of evidence in classical sources, this text also shows the difficulty of reconstituting a religious topography for this city, notably during the pre-Roman era. 
Mactaris/Maktar est une ville d'origine numide située actuellement dans la région du Haut-tell tunisien à $170 \mathrm{~km}$ au sud-ouest de Carthage. Son histoire remonte au milieu du $\mathrm{III}^{\mathrm{e}}$ siècle avant J.-C. ${ }^{1}$. Elle est alors rattachée au territoire carthaginois avant d'être reprise, un siècle plus tard, par le roi Massinissa. En 46 avant J.-C., elle passe sous la domination romaine, suite à l'annexion du royaume de Juba I et à sa transformation en provincia Africa nova.

Au plan de la géographie historique, elle fait partie de la chora Thusca numide, devenue pagus Thuscae sous les Romains ${ }^{2}$. Après la promotion de Zama Regia/Henchir Jama au statut de civitas libera par un bienfait de Jules César, Mactaris lui succède dans le rôle de chef-lieu du district administratif et financier de la Thusca, qui compte une cinquantaine de cités et auquel sera rattaché, au plus tard à partir de 113 après J.-C., le pagus Gunzuzi voisin qui en regroupe quatorze ${ }^{3}$.

Vers la fin du règne d'Auguste, entre 6 et 14 après J.-C., elle devient une station importante sur la voie stratégique aménagée par la Troisième Légion Auguste pour relier la capitale de la province d'Afrique proconsulaire, Carthage, à la nouvelle base de l'armée, Ammaedara/Haïdra 4 .

Hormis les nécropoles mégalithiques de la ville et quelques tronçons de sa muraille numide, aucun vestige archéologique ne

1. Mounira Harbi-Riahi, Abderrazak Gragueb, Gabriel Camps, Ali M'timet, Jamel Zoughlami, Atlas préhistorique de la Tunisie, feuille VIII : Mactar, Rome, École française de Rome, 1985, p. 34 : le mobilier funéraire trouvé à l'intérieur des tombes mégalithiques faisant partie de la nécropole sud de la ville date de la période qui s'étend entre le IV et le II ${ }^{\mathrm{e}}$ siècle avant J.-C.

2. Il s'agit d'une circonscription administrative remontant à l'époque punique et connue dans les sources classiques par un passage d'Appien (Libyca, 68-69). Elle est également citée dans des textes épigraphiques néo-puniques et latins. Sur cette circonscription, voir Gilbert-Charles Picard, Amar Mahjoubi, Azedine Beschaouch, "Pagus Thuscae et Gunzuzi », Comptes rendus des séances de l'Académie des Inscriptions et Belles-Lettres, 1963, p. 124-130; Ahmed M'charek, "De Zama à Kairouan : la Thusca et la Gamonia ", in : Frontières et limites géographiques de l'Afrique du Nord antique. Hommage à Pierre Salama, Paris, Publications de la Sorbonne, 1999, p. 139-183.

3. A. M'charek, art. cité, p. 148-149, notes 39 et 40.

4. Sur le réseau routier de la ville de Maktar, voir Ahmed M'charek, «Un itinéraire inédit dans la région de Maktar : tronçon de la voie augustéenne Carthage Ammaedara ", Bulletin archéologique du Comité des Travaux Historiques et Scientifiques. Fasc. B, Afrique du Nord, n.s. 22/1989, p. 153-167. 
remonte aux premiers siècles de la vie de Mactaris. Toutefois, quatorze inscriptions libyques et trois cents inscriptions néo-puniques publiées sont susceptibles de fournir des indications sur la cité et sa culture néo-punique ${ }^{5}$. Une place dallée aménagée au cœur de la partie ouest du site, couvrant $2400 \mathrm{~m}^{2}$, de forme irrégulière et dépourvue de portique, aurait servi de centre civique jusqu'au début du $\mathrm{II}^{\mathrm{e}}$ siècle après J.-C. C'est cette place que G.-Ch. Picard appelle le vieux forum ${ }^{6}$.

Il apparaît ainsi que le paysage religieux de Mactaris à l'époque préromaine est insuffisamment documenté. Pour essayer de le reconstituer, on ne dispose en effet, en l'absence de sources littéraires, que de rares informations sur les lieux de cultes numides. Ces informations nous sont fournies par des découvertes fortuites ou par les résultats des travaux de dégagements et de fouilles consacrés à certains monuments de la ville. Ces travaux ont permis la mise au jour d'un matériel archéologique varié (inscriptions, stèles, éléments d'architecture, céramique...) attestant la présence dans la ville de Mactaris d'édifices religieux remontant à l'époque numide. Toutefois, l'interprétation de ces découvertes pose parfois beaucoup plus de problèmes qu'elle n'en résout.

Trois lieux de culte semblent remonter à l'époque préromaine :

\section{Le sanctuaire de Hoter Miskar}

Un sanctuaire consacré à Hoter Miskar $^{7}$ est aménagé sur une crête située au nord-est du «vieux» forum. Repéré par

5. Une centaine d'inscriptions néo-puniques, découvertes en 1969 dans les fondations de l'arc de Bab el Aïn, sont encore inédites. Étudiées depuis les années 1970 par M'hamed Hassine Fantar et Maurice Sznycer, elles devraient en principe faire l'objet d'une publication par l'École française de Rome.

6. Gilbert-Charles Picard, «Les places publiques et le statut municipal de Mactar », Comptes Rendus des séances de l'Académie des Inscriptions et BellesLettres [dorénavant CRAI] 97/1953, p. 80-82.

7. Hoter Miskar est une divinité dont le nom n'apparaît en dehors de Maktar que sur quelques inscriptions puniques de Carthage. La nature et les fonctions de ce dieu, sans doute d'origine orientale, posent encore problème. Sur cette figure divine, cf. Edward Lipinski, Dieux et déesses de l'univers phénicien et punique [Studia Phoenicia 14], Leuven, Peeters, 1995, p. 174-176; Sergio Ribichini, "Mythes et rites des Phéniciens et des Carthaginois », in : Gregorio Del Olmo Lete (éd.), Mythologie et Religion des Sémites occidentaux, vol. II, Leuven, Peeters, 2008, p. 302. 
M. Delherbe à la fin du XIX ${ }^{\mathrm{e}}$ siècle $^{8}$, ce lieu sacré a fait l'objet de plusieurs fouilles qui ont permis le dégagement de nombreuses structures ayant appartenu à ce sanctuaire et aux édifices qui l'ont succédé9.

La confrontation des renseignements - fournis par le dossier épigraphique relatif à ce monument ${ }^{10}$ - aux données archéologiques permet de distinguer plusieurs phases dans la vie de ce complexe religieux. Durant la première phase, qui remonte au moins au début $\mathrm{du} \mathrm{I}^{\mathrm{er}}$ siècle avant J.-C., ce lieu sacré était composé d'un autel monumental qui s'élevait au milieu d'une aire à ciel ouvert ${ }^{11}$.

De part et d'autre de cet autel ont été élevés, à des dates différentes, plusieurs bâtiments. La première campagne de construction paraît être commémorée par une inscription néo-punique qui indique que quatre personnages ont édifié un temple à Hoter Miskar dans l'enceinte du lieu sacré ${ }^{12}$.

Au cours de la troisième phase, l'édifice a connu une importante transformation avec la construction d'un temple somptueux comportant une crypte sacrée, des frontons magnifiques et une chapelle. Ces travaux ont été financés par une association nommée Mizrah ${ }^{13}$

8. René Cagnat, Paul Gauckler, Les monuments historiques de la Tunisie, I: Les monuments antiques. Les temples païens, Paris, E. Leroux, 1898, p. 129-131.

9. Les premières fouilles menées sous la direction du capitaine Bordier, contrôleur civil à Maktar à la fin du XIXe siècle, ont permis de déblayer quelques structures. À partir de 1971, une équipe tuniso-française placée sous la direction de A. Beschaouch, de M.-H. Fantar et de G.-Ch. Picard a entrepris plusieurs campagnes de fouilles qui ont exhumé un complexe religieux constitué de plusieurs bâtiments construits à des dates différentes.

10. Le dossier épigraphique relatif à ce lieu sacré compte cinq inscriptions puniques et néo-puniques.

11. Sur cet autel, cf. Colette Picard, «Le grand autel du sanctuaire d'Hoter Miskar », Karthago XX/1982, p. 71-74; Gilbert-Charles Picard, "Le temple de Hoter Miskar à Mactar », Bulletin archéologique du C.T.H.S. Fasc. B, Afrique $d u$ Nord, n.s. 18/1988, p. 21-25. Selon les auteurs, l'autel avait des dimensions assez importantes avoisinant $10 \mathrm{~m}$ de longueur et plus de $5 \mathrm{~m}$ de large. Durant des dizaines d'années on a laissé s'accumuler les cendres en couches stratifiées autour de cet autel.

12. James-Germain Février, M'hamed Hassine Fantar, «Les nouvelles inscriptions monumentales néopuniques de Mactar», Karthago XII/1965, p. 58. Selon les auteurs, trois des quatre personnages nommés dans l'inscription portent des noms puniques. Leur acte n'a aucun caractère officiel. Il relève de particuliers largement influencés par la culture punique qui ont construit un sanctuaire dans un lieu où il n'y avait aucune bâtisse.

13. Plusieurs spécialistes voyaient dans le Mizrah une association cultuelle qui s'occupe de la construction et de l'entretien d'édifices religieux traditionnels, 
dont les membres ont été énumérés sur une grande inscription commémorative datable, selon J.-G. Février, des années 50-55 après J.-C. ${ }^{14}$.

Aucune trace de ces constructions n'a été trouvée lors des fouilles. Toutefois, entre la deuxième moitié du $\mathrm{I}^{\mathrm{er}}$ siècle après J.-C. et la fin

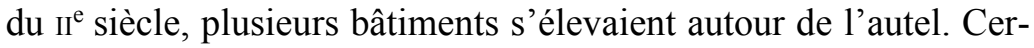
tains furent probablement des chapelles qui abritaient les images du maître du sanctuaire et de ses parèdres ${ }^{15}$.

À l'époque sévérienne, un nouveau temple à plan barlong est bâti. Il occupait la partie sud-ouest du téménos. Une autre partie du terrain sacré fut privatisée au profit d'une grande famille mactaroise qui y construit une somptueuse demeure ${ }^{16}$.

Dans la seconde moitié $d u \mathrm{IV}^{\mathrm{e}}$ siècle, le temple à plan barlong est converti en église sans modifications architecturales comme l'attestent les tombes établies sous les portiques et dans la cour ${ }^{17}$.

Au terme de plusieurs campagnes de fouilles, G.-Ch. Picard a conclu qu'à l'époque numide ce lieu sacré était composé d'une aire sacrée ayant pour centre un autel monumental dépourvu de décor architectonique. Le dieu Hoter Miskar était honoré par des sacrifices dont les restes, gardés sur place, se sont accumulés au fil des

notamment à l'époque romaine. Sur cette association, cf. Charles ClermontGanneau, "Le mazrah et les curiae, collegia ou ordines carthaginois dans le tarif des sacrifices de Marseille et dans les inscriptions néo-puniques de Maktar et d'Althiburos », CRAI 26/1898, p. 348-368; Gilbert-Charles Picard, "Civitas Mactaritana », Karthago VIII/1957, p. 62-63; Maurice Sznycer, « Quelques observations sur la grande inscription dédicatoire de Mactar », Semitica XXII/1972, p. 36-39; Alia Krandel Ben Younès, La présence punique en pays numide, Tunis, Institut national du patrimoine, 2002, p. 443.

14. James-Germain Février, "La grande inscription dédicatoire de Mactar», Semitica VI/1956, p. 15-31. Les lectures de ce savant ont été en partie critiquées une quinzaine d'années plus tard par M. Sznycer, «Quelques observations sur la grande inscription dédicatoire de Mactar», art. cité, p. 25-43.

15. G.-Ch. Picard, «Le temple de Hoter Miskar à Mactar», art. cité, p. 24; Idem, « Les chapelles », Karthago XX/1982, p. 82. L'auteur présente une description de ces fragments : l'un d'eux, appartenant très probablement à une statuette féminine mesurant $60 \mathrm{~cm}$ de hauteur, représente une main tenant l'extrémité inférieure d'une corne d'abondance. Un autre est un fragment d'épaule cuirassée qui devait appartenir à une statuette mesurant à peu près $60 \mathrm{~cm}$ de hauteur et qui paraît figurer le dieu Mars.

16. G.-Ch. Picard, « Le temple de Hoter Miskar à Mactar », art. cité, p. 21-24.

17. Gilbert-Charles Picard, «Essai d'interprétation du sanctuaire de Hoter Miskar à Mactar », Bulletin archéologique du C.T.H.S. Fasc. B, Afrique du Nord, n.s. $18 / 1988$, p. 17. 
années, formant des couches superposées de terre cendreuse parsemée de charbon, d'os et de céramique ${ }^{18}$.

Il est à noter qu'au début de son histoire la configuration de ce lieu de culte n'a rien de particulier. D'autres sanctuaires en Afrique du Nord sont ordonnés à peu près selon le même schéma; ainsi celui de Baal Hammon et de Tanit qui s'élevait à Bir Bouregba, l'antique Thinissut ${ }^{19}$, et celui de Baal Hammon et de Tanit à El Kenissia, situé à six km au sud-ouest de Sousse ${ }^{20}$. Les deux lieux comportaient une cour à ciel ouvert abritant un édicule ou un socle autour duquel ont été bâtis, durant des campagnes successives, plusieurs bâtiments. Certains furent des chapelles dans lesquelles sont déposées des figurines en terre cuite représentant les dieux du sanctuaire et leurs parèdres. L'originalité du sanctuaire de Hoter Miskar à Maktar réside dans les dimensions monumentales de l'autel qui pourraient être en corrélation avec la nature des sacrifices offerts à cette divinité.

\section{LE SANCTUAIRE SUBURBAIN}

Un sanctuaire anonyme s'élevait à l'ouest du « vieux » forum, à la limite des ruines connues de la ville. Il est attesté uniquement

18. C. Bourgois, «Compte rendu sommaire de la dernière campagne de fouilles à Mactar (Tunisie) », Bulletin archéologique du C.T.H.S. Fasc. B, Afrique du Nord, n.s. 18/1988, p. 10.

19. Concernant ce lieu de culte, voir Hédi Dridi, Meriem Sebaï, «De Tenesmat à Thinissut, nouvelles observations sur l'aménagement d'un lieu de culte africain », in: Lieux de cultes : aires votives, temples, églises, mosquées. $I X^{e}$ colloque international sur l'histoire et l'archéologie de l'Afrique du Nord antique et médiévale, Tripoli, 19-25 février 2005, Paris, CNRS Éditions, 2008, p. 101-118. Les auteurs ont distingué cinq phases dans la vie du complexe religieux de Thinissut. Durant la plus ancienne, qui remonte à l'époque punique, a été construit « un sanctuaire régulier et symétrique, composé d'une cour quadrangulaire portiquée et d'un édicule placé sur son axe longitudinal ».

20. Ce sanctuaire a fait l'objet d'une fouille au début du $\mathrm{Xx}^{\mathrm{e}}$ siècle sous la direction de Louis Carton: «Le sanctuaire de Tanit à El-Kénissia », Mémoires présentés par divers savants à l'Académie des Inscriptions et Belles-Lettres, 1908, p. 1-160. Le plan du sanctuaire publié par L. Carton est relatif à la phase tardive de ce lieu de culte. Au début de son histoire, ce lieu sacré était composé d'une cour à portique au milieu de la quelle se dressait un socle à escalier : Alexandre Lézine, "Résistance à l'hellénisme de l'architecture religieuse de Carthage », Les cahiers de Tunisie 26-27/1959, p. 258. 
par une inscription néo-punique mutilée, mentionnant un "prêtre » et une « chambre auprès du temple ». Selon J.-G. Février, celle-ci pourrait être une grande salle consacrée au banquet sacrificiel après l'immolation de la victime ${ }^{21}$. Nous ignorons le plan de ce lieu sacré et la date de sa construction. À l'époque romaine, probablement sous le règne d'Hadrien, un grand temple fut élevé dans ce lieu. Il était consacré à Apollon, l'un des dii patrii de Mactaris ${ }^{22}$.

\section{L'AIRE SACRÉE de BAAL HAMMon}

Ce lieu sacré, attesté depuis le début du XIX ${ }^{\mathrm{e}}$ siècle, pose encore aujourd'hui deux problèmes majeurs: le premier concerne son emplacement qui n'est pas identifié avec certitude et le deuxième son histoire, difficile à reconstituer dans l'état actuel de la documentation.

Cette aire est attestée uniquement par de nombreux ex-voto trouvés à Maktar depuis le début du XIX ${ }^{\mathrm{e}}$ siècle. Si l'emplacement de ce lieu sacré pose encore problème, c'est parce que d'une part, ces monuments n'étaient pas en place lors de leur découverte et, d'autre part, il manque un élément décisif pour cette identification, à savoir les urnes cinéraires. En effet, l'un des rituels fondamentaux dans le culte de Baal Hammon est le passage par le feu des victimes offertes au dieu. Une fois consumées par le feu, les cendres des organes concernés sont ramassées et mises dans des urnes destinées à être enterrées dans le sanctuaire même. C'est la découverte de ces urnes, dont certaines contenaient des restes humains calcinés, qui explique le nom de « tophets » donné par les premiers spécialistes de la religion punique aux lieux de culte relatifs à cette divinité2 ${ }^{23}$. Ce terme désigne généralement une aire sacrée à ciel ouvert qui se présente comme un champ d'urnes contenant parfois les ossements calcinés d'enfants en bas âge et de petits animaux. Ces urnes sont

21. James-Germain Février, «Sur trois inscriptions néopuniques trouvées à Maktar », BCTH 1950, p. 111-112.

22. G.-Ch. Picard, « Civitas Mactaritana », art. cité, p. 150.

23. Edward Lipinski (dir.), Dictionnaire de la civilisation phénicienne et punique, Turnhout, Brepols, 1992: le mot tophet est emprunté à l'Ancien Testament et désigne l'endroit de la vallée de Ben Hinnom, à la périphérie de Jérusalem, où l'on offrait des enfants en sacrifices. 
souvent surmontées de stèles portant divers motifs et parfois une inscription dédicatoire ${ }^{24}$.

Malgré l'absence d'urnes sacrificielles, une première hypothèse d'identification de cette aire a été émise par G.-Ch. Picard qui, en se fondant sur un lot ancien de stèles votives trouvées aux confins de la nécropole nord, a supposé que « le tophet de Mactar se trouvait sur les pentes du ravin d'Aïn el $\mathrm{Bab}^{25} »$. L'absence d'urnes est justifiée, selon l'auteur, par l'érosion de l'oued qui a pu bouleverser l'aire sacrée et faire disparaître le mobilier votif qui y était déposé. Mais cette hypothèse ne résiste pas à la critique, car les ex-voto trouvés à cet endroit n'étaient pas en place. La découverte d'un second lot de stèles à Baal Hammon en 1969, lors de la restauration de Bab el Aïn, a montré que les stèles en question étaient en fait réemployées dans les soubassements de l'arc construit à l'entrée nord de la ville et dans la terrasse qui le supportait ${ }^{26}$.

Une deuxième hypothèse d'identification du tophet de Maktar a été émise par A. M'charek : ce lieu sacré, qui ne peut pas être situé au fond du ravin de Bab el Aïn, devrait être recherché audessous du sanctuaire dédié à Saturne, converti en église dite de Rutilius $^{27}$. Placer l'aire sacrée de Baal Hammon sous le temple de Saturne est une hypothèse qui se défend à plusieurs titres. En effet, en Afrique du Nord, Saturne était l'héritier de Baal Hammon, et de nombreux sanctuaires traditionnels furent remplacés au II $^{\mathrm{e}}$ ou au début $\mathrm{du} \mathrm{III}^{\mathrm{e}}$ siècle par des temples construits à la romaine. Dans

24. S. Ribichini, "Mythes et rites des Phéniciens et des Carthaginois », op. cit., p. 345-346.

25. G.-Ch. Picard, « Civitas Mactaritana », art. cité, p. 43.

26. Au cours de la discussion qui a suivi la communication d'Hélène Bénichou-Safar sur les tophets et nécropoles puniques (présentée au colloque consacré aux Monuments funéraires et institutions autochtones en Afrique du Nord antique et médiévale, tenu à Pau en 1993 et publié par le CTHS en 1995, p. 91-102), A. M'charek a évoqué le tophet de Maktar et les stèles votives qui y ont été déposées, en affirmant que " tous les ex-voto récoltés autour de l'arc de Bab el Aïn sont des fragments cassés et déplacés pour être remployées dans la fondation du même arc et de la terrasse qui le supportait $»$.

27. Ahmed M'charek, «La romanisation du culte de Ba'al Hammon dans la région de Maktar (antique Thusca) », in : M'hamed Hassine Fantar, Mansour Ghaki (éd.), Actes du III Congrès international des études phéniciennes et puniques, Tunis 11-16 novembre 1991, Tunis, Institut national du patrimoine, 1995, vol. II, p. 245-247. 
plusieurs cas, le mobilier votif déposé à l'intérieur de l'aire sacrée est ramassé et entassé dans une ou plusieurs favissae. Parmi les exemples les mieux connus en Proconsulaire nous pouvons citer ceux de Thugga ${ }^{28}$ et de Thuburnica ${ }^{29}$.

Les différents travaux de dégagement menés dans ladite église ont permis de constater qu'elle couvrait un des plus grands temples de la ville de Maktar ${ }^{30}$. L'étude de N. Duval consacrée à ce monument a permis de reconstituer son plan. Selon ce savant, le temple occupait une plate-forme de 45,20 m sur 20,75 m. On y accédait par un escalier monumental de sept marches, bordé des deux côtés par deux pièces de plan carré dont seule celle qui se trouve à gauche est encore conservée - l'autre se trouvant occupée par l'actuel musée du site. Cette entrée permettait d'accéder à une cour à ciel ouvert bordée d'un triple portique. Les portiques est et ouest se terminent au fond par une exèdre. Trois cellae occupent le fond de la cour ${ }^{31}$.

L'attribution à Saturne de ce temple, construit vers 210, résulte de deux indices : le fait qu'il soit à proximité de l'endroit présumé du tophet - c'est-à-dire du ravin de Bab el Aïn, où furent découvertes beaucoup de stèles consacrées à Baal Hammon -, et que son plan, comportant une cour et trois cellae, soit comparable à celui de

28. Depuis sa découverte à la fin $\mathrm{du} \mathrm{XIX}^{\mathrm{e}}$ siècle, le sanctuaire de Saturne à Dougga a fait l'objet de plusieurs campagnes de fouilles qui ont permis de reconstituer son plan et de fixer la date de sa construction. Ses travaux ont également permis la mise au jour d'un mobilier votif varié relatif à Baal Hammon, constitué essentiellement de stèles et de poteries enterrées dans des favissae aménagées dans la cour du sanctuaire. Sur ce lieu sacré, cf. Marcel Le Glay, Saturne africain. Monuments, t. I, Paris 1961, p. 208-212; Patrizio Pensabene, « Il tempio di Saturni a Dougga e tradizioni architettoniche di origine punica », L'Africa romana, VII/1989, p. 251-293; Sophie Saint-Amans, Topographie religieuse de Thugga (Dougga). Ville romaine d'Afrique proconsulaire (Tunisie), Bordeaux, Ausonius, 2004, p. 348-357.

29. Ce temple a été fouillé en 1906 par L. Carton. Depuis, il n'a fait l'objet d'aucune étude systématique. Nous savons que la fouille a permis la découverte d'un mobilier votif relatif à la phase préromaine du culte. À une date indéterminée, un sanctuaire à la romaine - composé d'une cour à colonnades au fond de laquelle s'élevaient trois cellae précédées d'un vestibule - a été élevé en marge d'une area sacrée. Sur ce sanctuaire, voir M. Le Glay, Saturne africain. Monuments, op. cit., p. $274-277$.

30. Gilbert-Charles Picard, «Le temple du musée à Mactar », Revue archéologique I/1984, p. 13-28.

31. Noël Duval, «une hypothèse sur la basilique de Rutilius à Mactar», Revue des Études Augustiniennes, 31/1985, p. 30-37. 
plusieurs temples africains consacrés à Saturne, à l'instar de ceux de Thugga ${ }^{32}$, d'Ammaedara ${ }^{33}$ et de Thamugadi ${ }^{34}$.

Mais cette attribution à Saturne, fort plausible, demeure incertaine. En effet, dans l'état actuel des recherches, ce supposé temple à Saturne n'a livré ni urnes cinéraires, ni stèles. Les multiples travaux de dégagement qu'a connu cet édifice depuis la fin du XIX ${ }^{\mathrm{e}}$ siècle n'ont abouti qu'à l'enlèvement des couches de remblai qui le couvraient, et à la découverte, dans les déblaiements, de plusieurs inscriptions néo-puniques et latines, réemployées dans les murs de la basilique qui a succédé au temple païen ${ }^{35}$.

Nous pensons que l'aire sacrée de Baal Hammon devait se trouver à la périphérie du périmètre urbain, lieu d'emplacement fréquemment choisi pour l'aménagement des lieux de culte de cette divinité en Afrique du Nord. À Maktar, cette aire est très probablement située non loin de l'arc de Septime Sévère, élevé à l'entrée nord de la ville, étant donné qu'un très grand nombre de stèles étaient

32. Concernant le plan de ce temple, cf. supra note 28.

33. Le temple de Saturne d'Ammaedara a été identifié par L. Poinssot et dégagé, à partir de 1930, sous la responsabilité de G. Dolcemascolo (médecin de la mine des phosphates de Kaala Jerda). Cependant, les résultats de ces travaux n'ont jamais fait l'objet d'une publication. Dans son étude consacrée au Saturne africain, M. Le Glay a publié un croquis figurant le plan de ce temple en précisant qu'il «n'est pas coté et [que] les proportions des différentes parties ne sont sans doute pas rigoureusement exactes ». Toutefois, la figure 6 permet d'avoir une idée sur les composantes de ce monument : M. Le Glay, Saturne africain. Monuments, op. cit., t. I, p. 324. La publication de nouveaux documents lapidaires provenant de ce temple a été accompagnée d'une description détaillée de l'état de conservation de ses ruines en 2000 : François Baratte, Zeïneb Benzina Ben Abdallah, «Le sanctuaire de Saturne à Ammaedara (Haödra, Tunisie) », Revue Archéologique 1/2000, p. 54-56.

34. Ce temple, repéré et en partie dégagé en 1921 par Ch. Godet, a été fouillé à partir de 1955 sous la direction de M. Le Glay qui nous a laissé une étude détaillée sur ce temple dans le tome II de son ouvrage Saturne africain. Monuments, op. cit., p. 126-129.

35. Depuis la découverte de l'épitaphe de l'évêque Rutilius, de nombreux travaux de dégagement ont été entrepris dans le but de trouver de nouvelles inscriptions. D'autres épitaphes chrétiennes ont été découvertes durant l'hiver 1883-1884 : Françoise Prévot, Recherches archéologiques franco-tunisiennes à Mactar, V. Les inscriptions chrétiennes, Rome, 1984, p. 11-18. Quelques années plus tard, le capitaine Bordier a exhumé dans les ruines de l'église une série de bases de statues d'empereurs du IV siècle (R. Cagnat, «Inscriptions nouvelles d'Afrique », $B C T H$ 1889, p. 362-364; CIL VIII, 11804-11809); un autel criobolique et taurobolique datable du règne de Probus (CIL VIII, 23400) ainsi qu'une colonne votive à Liber Pater avec l'album du corpus fullonum (CIL VIII, 23399=ILS 3362, ILPB, $\mathrm{n}^{\circ} 61$ ). De cette église provient l'une des deux grandes inscriptions dédicatoires du temple de Hoter Miskar. 
réemployées dans ces environs immédiats. Mais, elle ne peut pas se confondre avec la nécropole qui s'étendait au nord de cet arc. Elle ne peut pas non plus être aménagée au fond du ravin qui longeait l'arc car les aires sacrées de Baal Hammon occupaient souvent des sites de hauteur ${ }^{36}$. Elle est à rechercher plutôt sur la rive droite du ravin de Bab el Aïn, c'est-à-dire sur les hauteurs qui se trouvent au sud de ce dernier. La zone qui se trouvait entre l'actuelle maison des fouilles du site et la basilique dite de Rutilius pourrait constituer l'area dans laquelle furent déposés les ex-voto. À l'instar de celui de Thuburnica, le temple pourrait être construit au-delà de l'aire où furent déposées les urnes et les stèles qui les surmontent. Des sondages pourraient confirmer ou infirmer notre hypothèse.

Le seul témoignage archéologique relatif à cette aire consiste en deux cents stèles, découvertes hors contexte stratigraphique. La première série d'ex-voto consacrée à Baal Hammon a été trouvée au début du XIX ${ }^{\mathrm{e}}$ siècle, mais aucun détail concernant les conditions et l'endroit précis de la découverte ne fut donné à ces stèles lors de leur publication ${ }^{37}$. Certaines de ces stèles sont soit perdues, soit conservées dans des musées européens.

En 1887, le capitaine D. Bordier, premier contrôleur civil à Maktar, découvrit lors de la construction de sa résidence dans la ville de nouvelles stèles épigraphes dont l'une était dédiée à Baal Hammon ${ }^{38}$. Encouragé par M. Renan, Bordier s'est mis à la recherche de nouvelles inscriptions, et c'est en 1889 qu'il a pu exhumer un lot de 63 ex-voto épigraphes au pied de la porte triomphale, près de la source d'Aïn el $\mathrm{Bab}^{39}$. En 1950, de nouvelles stèles furent découvertes loin de l'arc de Bab el Aïn, dans un endroit situé sur le decumanus maximus desservant la place sévérienne située au sud du nouveau forum ${ }^{40}$. Conservés au Musée Alaoui, ces monuments ont été inventoriés par C. Picard. Une brève description iconogra-

36. Marcel Le Glay, Saturne africain Histoire, Paris 1966, p. 270. Selon l'auteur, le choix d'un haut lieu pour aménager un sanctuaire à Baal/Saturne correspond parfaitement à la conception ouranienne et transcendantale de ce dieu.

37. Quelques-unes furent publiées par l'abbé François Bourgade : Toison d'or de la langue phénicienne, Paris $1856^{2}$.

38. Philippe Berger, "Inscriptions libyques et néo-puniques provenant de Mactar », CRAI 18/1889, p. 99.

39. Idem, «Les inscriptions de Maktar », CRAI 19/1890, p. 36.

40. Gilbert-Charles Picard, « Mactar», BCTH 1951-1952, p. 98-100. 
phique accompagnait les notices de ces stèles ${ }^{41}$ et fut reprise par le même auteur dans une communication présentée en 1987 au Congrès des études phéniciennes et puniques ${ }^{42}$.

Enfin un dernier lot comptant une centaine de stèles inédites a été trouvé fortuitement en 1969, au cours des travaux de renforcement de l'arc de triomphe de Bab el Aïn; elles étaient remployées depuis l'Antiquité dans le soubassement de l'arc.

L'étude des caractéristiques techniques et stylistiques de ces stèles et de l'évolution de leur programme iconographique pourrait permettre d'éclairer quelques épisodes de l'histoire de cette aire sacrée. L'examen du répertoire iconographique des stèles néopuniques permet de distinguer trois groupes.

Le premier groupe est constitué de stèles figurant des motifs religieux qui dérivent de prototypes puniques tels que le signe dit de Tanit et le caducée. Le dieu est invoqué par un motif qui consiste en un visage humain entouré de rayons solaires et surmonté - ou surmontant - un croissant lunaire aux pointes tournées vers le haut. Les personnages figurés sur les monuments de ce groupe sont traités dans un style très schématisé. Vêtus d'une courte tunique numide, ils étendent les bras qui brandissent de longues tiges ornées de grenades ou de feuilles. Une dédicace gravée en caractères néopuniques occupe le milieu de la stèle.

Le deuxième groupe est constitué de stèles qui portent un décor plus chargé : au lieu du signe dit de Tanit, d'autres motifs ornent la face antérieure de ces monuments. Au fronton, à côté du visage radié, remplacé parfois par une rosace ou un croissant, apparaissent des poissons et des oiseaux. Les dédicants sont figurés à l'intérieur d'une niche évidée; leurs traits s'humanisent et les femmes se parent de bijoux. Il convient de souligner que ces stèles sont exécutées dans un style qui ressemble beaucoup à celui des stèles funéraires numides, datables de la période d'Auguste à la fin du règne de $\operatorname{Trajan}^{43}$, ce qui laisse supposer que ces ex-voto étaient produits dans les mêmes ateliers et dateraient de la même période.

41. Colette Picard, Catalogue du Musée Alaoui. Nouvelle série, Collections puniques, 2 vol., Tunis 1954, p. 273.

42. Id., «Les Maktarois vus par eux-mêmes entre la conquête césarienne et la promotion coloniale », Atti del II Congresso Internazionale di Studi Fenici e Punici (Roma, 9-14 novembre 1987), Roma, CNR, 1991, t. I, p. 193-198.

43. Ahmed M'charek, Aspects de l'évolution démographique et sociale à Mactaris, Tunis, 1982, p. 18-45. 
Le troisième groupe est constitué de fragments de stèles qui offrent de nombreuses analogies avec les stèles dites de la Ghorfa provenant de Maghrawa, un vicus situé sur le territoire de Mactaris ${ }^{44}$. Ces monuments se caractérisent par un programme iconographique propre, marqué essentiellement par l'anthropomorphisation des assesseurs du grand dieu Baal Hammon, alors que ce dernier est encore suggéré par des symboles. Plusieurs études ont été consacrées à ce groupe de stèles et les spécialistes s'accordent pour les dater au plus tard du règne d'Hadrien ${ }^{45}$.

Dans l'état actuel des recherches, il semble que le tophet a été abandonné durant la deuxième moitié du $\mathrm{II}^{\mathrm{e}}$ siècle après J.-C. Cet abandon ne signifie pas que le culte de Baal Hammon a disparu. Il a été plutôt romanisé comme l'attestent deux documents trouvés dans les ruines de la ville. Le premier est une dédicace latine fragmentaire consacrée à Saturne pour le salut de l'empereur Septime Sévère ${ }^{46}$. Le second est un buste figurant un personnage masculin dans la force de l'âge, barbu, avec une chevelure fournie recouverte d'un voile $^{47}$. Selon M. Le Glay, ce buste de facture grossière est conforme au canon classique, ce qui permet de le dater du $\mathrm{II}^{\mathrm{e}}$ siècle $^{48}$.

En somme, nous pensons que Baal Hammon occupait une place importante dans le panthéon mactarois. Son culte, qui a connu son

44. Concernant Maghrawa, voir Ahmed M'charek, «Maghrawa, antique Macota (Tunisie) », Antiquités africaines 33/1997, p. 119-127. Dans une étude publiée dans les MEFRA, l'auteur a démontré que la fameuse collection de stèles dites de la Ghorfa provient du site de Maghrawa : id., " Maghrawa, lieu de provenance des stèles punico-numides dites de la Ghorfa ", Mélanges de l'École française de Rome. Antiquité, 100-2/1988, p. 731-760.

45. Les stèles dites de la Ghorfa, découvertes depuis le XIX ${ }^{\mathrm{e}}$ siècle, sont dispersées dans de nombreux musées. La première étude systématique qui fut consacrée à ces stèles est due à Anna Maria Bisi : «A proposito di alcune stele del tipo della Ghorfa al British Museum », Antiquités africaines 12/1978, p. 21-88. Une dizaine d'années plus tard, ce même groupe de stèles fut étudié par Elena Francesca Guedini, "Ancora sulle stele della Ghorfa. Qualche precisazione », L'Africa Romana VII/1989, p. 233-244 : selon l'auteur, le dieu auquel sont offerts ces ex-voto est Baal Hammon et non Caelestis. En ce qui concerne la datation de ce groupe de stèles, E. F. Guedini a proposé le règne d'Hadrien comme terminus post quem. D'autres études ont été consacrées au sujet : on retiendra notamment celle de Jennifer P. Moore, "Cultural elasticity in the inscriptions of so-called "la Ghorfa" stelae », Antiquités africaines 35/1999, p. 31-37.

46. CIL, 23403 : [Sat]urno Aug(usto) [sacr(um)/ pro sa]ute Im[p(eratoris) Caes(aris) L(ucii) Sep/timi S]eueri[---. L'inscription daterait des années 193-211.

47. Nous ignorons l'endroit exact de la découverte de ce buste.

48. M. Le Glay, Saturne africain, op. cit., 1961, p. 242, nº 1 . 
apogée durant le $\mathrm{I}^{\mathrm{er}}$ siècle et le premier quart du $\mathrm{II}^{\mathrm{e}}$ siècle après J.-C., était implanté dans la ville depuis l'époque préromaine. En effet, les motifs de tradition punique qui ornaient certaines stèles (signe dit de Tanit, caducées...) trahissent une conformité au discours religieux punique, caractérisé essentiellement par l'abstraction. D'autre part, le choix d'un endroit à la périphérie du périmètre urbain pour implanter l'aire sacrée de Baal Hammon reflète l'attachement des autorités mactaroises à une sorte de règle suivie par les collectivités dans la quasi-totalité des villes africaines.

En essayant de reconstituer le paysage religieux de Mactaris, nous avons rencontré plusieurs difficultés comme l'absence d'indications dans les sources littéraires, les problèmes d'interprétation dus aux conditions dans lesquelles furent menés les fouilles et les dégagements $\mathrm{du} \mathrm{XIX}^{\mathrm{e}}$ et du début du $\mathrm{XX}^{\mathrm{e}}$ siècle, sans compter les maigres informations dont nous disposons sur l'histoire de la ville de Mactaris, en particulier sur l'étendue du périmètre urbain et la répartition des édifices publics à l'intérieur de ce périmètre.

Or, si on admet, à la suite de G.-Ch. Picard, que le «vieux » forum constitue la première place publique de la cité, on constate que le temple de Hoter Miskar, l'aire sacrée de Baal Hammon et le temple anonyme qui a précédé celui d'Apollon n'occupaient pas le centre de la ville mais qu'ils avaient plutôt une situation périphérique.

Bien qu'elle soit située en pays numide, la ville de Maktar est nettement influencée par la civilisation punique. Cette influence est perceptible à travers l'usage de la langue phénicienne, l'adoption des institutions carthaginoises et des cultes puniques. En effet, Baal Hammon et Hoter Miskar sont deux divinités d'origine orientale. Leurs sanctuaires étaient desservis par un clergé dont la hiérarchie et les fonctions devaient être conformes au modèle oriental. Toutefois, dans l'état actuel des connaissances, il est très difficile d'identifier le dieu du temple suburbain et de reconstituer les rites pratiqués dans chacun des trois lieux sacrés ${ }^{49}$.

lamia.benabid@yahoo.fr

49. J'exprime mes plus vifs remerciements au professeur Ahmed M'charek qui a eu l'extrême amabilité de relire cet article : qu'il trouve ici l'expression de ma gratitude. J'adresse aussi mes remerciements à mon ami et collègue Mohamed Grira qui a réalisé les deux illustrations de cet article. 


\section{BIBLIOGRAPHIE}

François Baratte, Zeïneb Benzina Ben Abdallah, «Le sanctuaire de Saturne à Ammaedara (Haïdra, Tunisie) : documents inédits ", Revue Archéologique 1/2000, p. 51-75.

Philippe BERGER, «Inscriptions libyques et néo-puniques provenant de Mactar », Bulletin archéologique du Comité des travaux historiques et scientifiques [dorénavant : BCTH] 1889, p. 98-101.

Philippe Berger, "Les inscriptions de Mactar», Comptes rendus des séances de l'Académie des Inscriptions et Belles-Lettres [dorénavant: CRAI], 1890, p. 35-42.

Philippe BERGER, «Rapport sur sept nouvelles inscriptions néo-puniques et deux inscriptions libyques de Maktar », BAC 1890, p. 461-462.

Anna Maria Bisı, «A proposito di alcune stele del tipo della Ghorfa al British Museum », Antiquités Africaines 12/1978, p. 21-88.

C. Bourgois, «Compte rendu sommaire de la dernière campagne de fouilles à Mactar (Tunisie), $1982 », B C T H$, n.s., 1988, p. 7-15.

René Cagnat, Paul Gauckler, Les monuments historiques de la Tunisie, I : Les monuments antiques. Les temples païens, Paris, E. Leroux, 1898.

Louis CARTON, Le sanctuaire de Tanit à El-Kénissia, Mémoires présentés par divers savants à l'Académie des Inscriptions et Belles-Lettres, 1908, p. 1-160.

J.-B. Снавот, « Les inscriptions néo-puniques de Maktar », Journal Asiatique VII/1916, p. 87-103.

Hédi DRIDI, Meriem SÉBAÏ, « De Tanesmat à Thinissut. Nouvelles observations sur l'aménagement d'un lieu de culte africain ", in : Lieux de cultes : aires votives, temples, églises, mosquées. IX colloque international sur l'histoire et l'archéologie de l'Afrique du Nord antique et médiévale, Tripoli, 19 au 19 février 2005, Paris, CNRS Éditions, 2008, p. 101-117.

Noël Duval, «Une hypothèse sur la basilique de Rutilius à Mactar et le temple qui l'a précédé », Revue d'Études Augustiniennes 31/1985, p. $20-45$.

James-Germain FÉVRIER, «Trois inscriptions néo-puniques trouvées à Maktar », BCTH 1950, p. 111-113.

James-Germain FÉVRIER, « La grande inscription dédicatoire de Mactar », Semitica VI/1956, p. 15-31. 
James-Germain FÉVRIER, M'hamed Hassine FANTAR, « Les nouvelles inscriptions monumentales néo-puniques de Mactar », Karthago XII/1965, p. $45-59$.

Elena Francesca GuEdinI, «Ancora sulle stele della Ghorfa. Qualche precisazione », L'Africa Romana 7/1989, p. 233-244.

Mounira Harbi-Riahi, Abderrazak Gragueb, Gabriel CAmps, Ali M'timet et Jamel Zoughlami, Atlas Préhistorique de la Tunisie, VIII, Mactar, Rome, École française de Rome, 1985, p. 31-35.

Marcel Le Glay, Saturne africain. Monuments, 2 vol., Paris, 1961-1966.

Marcel Le Glay, Saturne africain. Histoire, Paris, 1966.

Alexandre LÉZINE, « Résistance à l'hellénisme de l'architecture religieuse de Carthage », Les cahiers de Tunisie 26-27/1959, p. 247-261.

Edward LIPINSKI, Dieux et déesses de l'univers phénicien et punique, Studia Phoenicia 14, Leuven, Peeters, 1995.

Ahmed M'chareK, Aspects de l'évolution démographique et sociale à Mactaris aux II et III $^{e}$ siècles après J.-C., Tunis 1982.

Ahmed M'CHAREK, « Documentation épigraphique et croissance urbaine : l'exemple de Mactaris aux trois premiers siècles de l'ère chrétienne », L'Africa romana 2/1984, p. 231-223.

Ahmed M'CHAREK, « Un itinéraire inédit dans la région de Maktar. Tronçon de la voie augustéenne Carthage - Ammaedara », Bulletin archéologique du Comité des Travaux Historiques et Scientifiques. Fasc. B, Afrique du Nord, n.s. 22/1987-1989, p. 153-167.

Ahmed M'CHAREK, «Maghrawa, lieu de provenance des stèles puniconumides dites de la Ghorfa », Mélanges de l'École française de Rome. Antiquité 100-2/1988, p. 731-760.

Ahmed M'chAReK, «La romanisation du culte de Ba'al Hammon dans la région de Maktar (antique Thusca) », in M'hamed Hassine Fantar, Mansour Ghaki (éd.), Actes du III Congrès international des études phéniciennes et puniques, Tunis, 11-16 novembre 1991, Tunis, Institut national du patrimoine, 1995, t. II, p. 245-257.

Ahmed M'charek, « Maghrawa, antique Macota (Tunisie)», Antiquités africaines 33/1997, p. 119-127.

Ahmed M'chAReK, « De Zama à Kairouan : la Thusca et la Gamonia », in : Frontières et limites géographiques de l'Afrique du Nord antique. Hommage à Pierre Salama, Paris, Publications de la Sorbonne, 1999, p. 139-183. 
Jennifer P. Moore, « Cultural elasticity in the inscriptions of so-called "la Ghorfa" stelae ", Antiquités africaines 35/1999, p. 31-37.

Patrizio Pensabene, « Il tempio di Saturno a Dougga e tradizioni architettoniche di origine punica », L'Africa Romana 7/1989, p. 251-294.

Colette Picard, Catalogue du Musée Alaoui. Nouvelle série, collections puniques, 2 vol., Tunis, 1954.

Colette PiCARD, « Le grand autel du sanctuaire d'Hother Miskar à Mactar », Karthago XX (1982), p. 71-74

Colette PiCARD, « Les Mactarois vus par eux-mêmes entre la conquête césarienne et la promotion coloniale ", Atti del II Congresso Internazionale di Studi Fenici e Punici (Roma, 9-14 novembre 1987), Roma, CNR, 1991, t. I, p. 193-198.

Gilbert-Charles PICARD, « Mactar », BCTH (1951-1952), p. 97-104.

Gilbert-Charles PiCARD, «Les places publiques et le statut municipal de Mactar ", Comptes Rendus des séances de l'Académie des Inscriptions et Belles-Lettres 97/1953, p. 80-82.

Gilbert-Charles PiCARD, Civitas Mactaritana, Karthago VIII/1957, p. 3-165.

Gilbert-Charles Picard, Amar Mahjoubi, Azedine Beschaouch, « Pagus Thuscae et Gunzuzi », CRAI 107/1963, p. 124-130.

Gilbert-Charles PiCARD, « Le temple du Musée à Mactar », Revue Archéologique 1/1984, p. 13-28.

Gilbert-Charles PiCARD, «Essai d'interprétation du sanctuaire de Hoter Miskar à Mactar », Bulletin archéologique du C.T.H.S. Fasc. B, Afrique $d u$ Nord, n.s. 18/1988, p. 17-20.

Gilbert-Charles PiCARD, «Le temple de Hoter Miskar à Mactar », BCTH n.s. 18 B (1988), p. 21-25. Idem

Françoise PRÉVOT, Recherches archéologiques franco-tunisiennes à Mactar, V. Les inscriptions chrétiennes, Rome, 1984.

Sergio Ribichini, « Mythes et rites des Phéniciens et des Carthaginois », in : Gregorio Del Olmo Lete (éd.), Mythologie et Religion des Sémites occidentaux, t. II, Leuven, Peeters, 2008.

Sophie SaInt-Amans, Topographie religieuse de Thugga (Dougga) ville romaine d'Afrique proconsulaire (Tunisie), Bordeaux, Ausonus, 2004.

Maurice SzNYCER, "Antiquités et épigraphie nord-sémitiques », Annuaire de l'École Pratique des Hautes Études, IV section - Sciences historiques et philologiques, 1977, p. 177-186. 


\section{ILLUSTRATIONS}

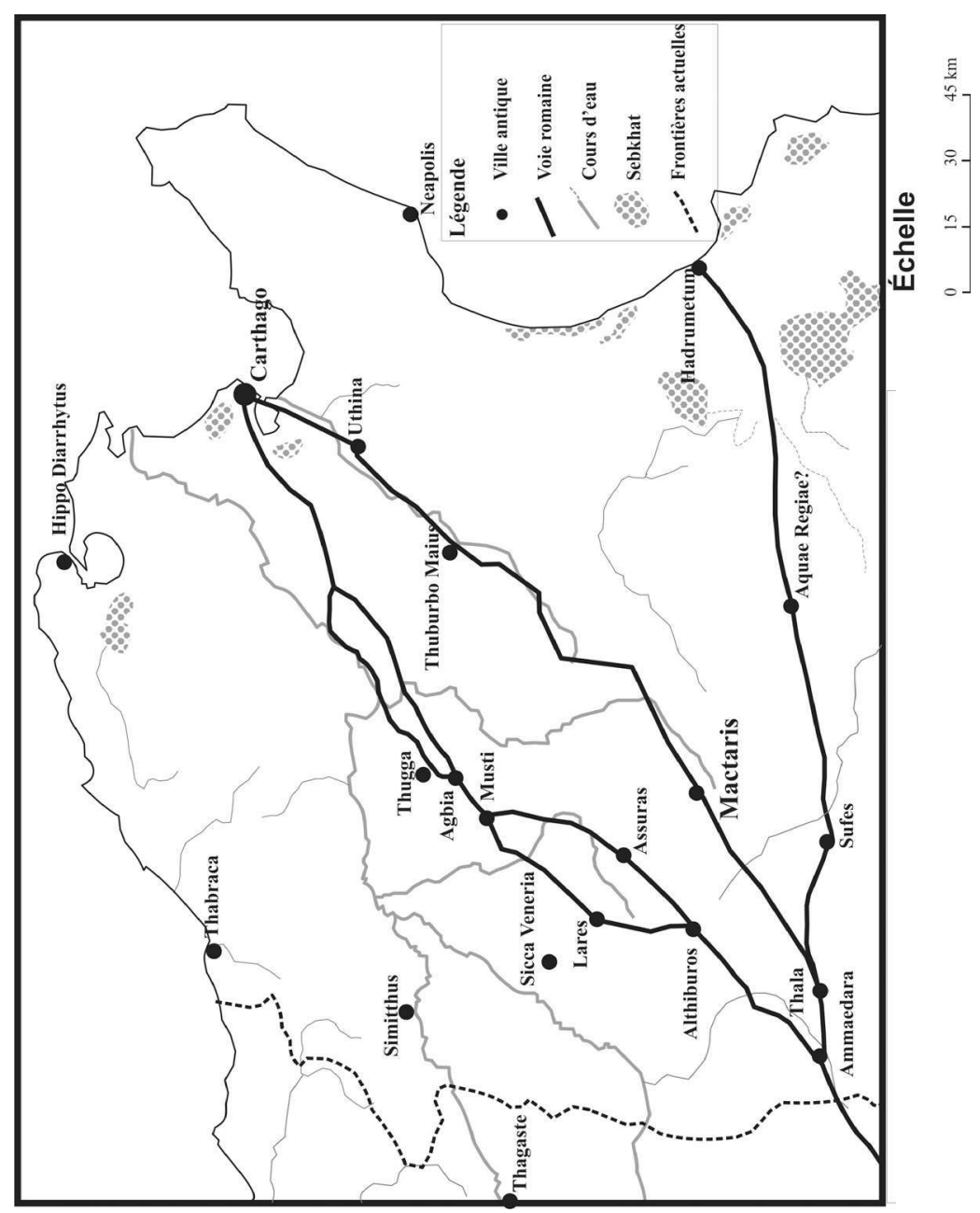

Fig. 1. Carte de localisation de Mactaris 


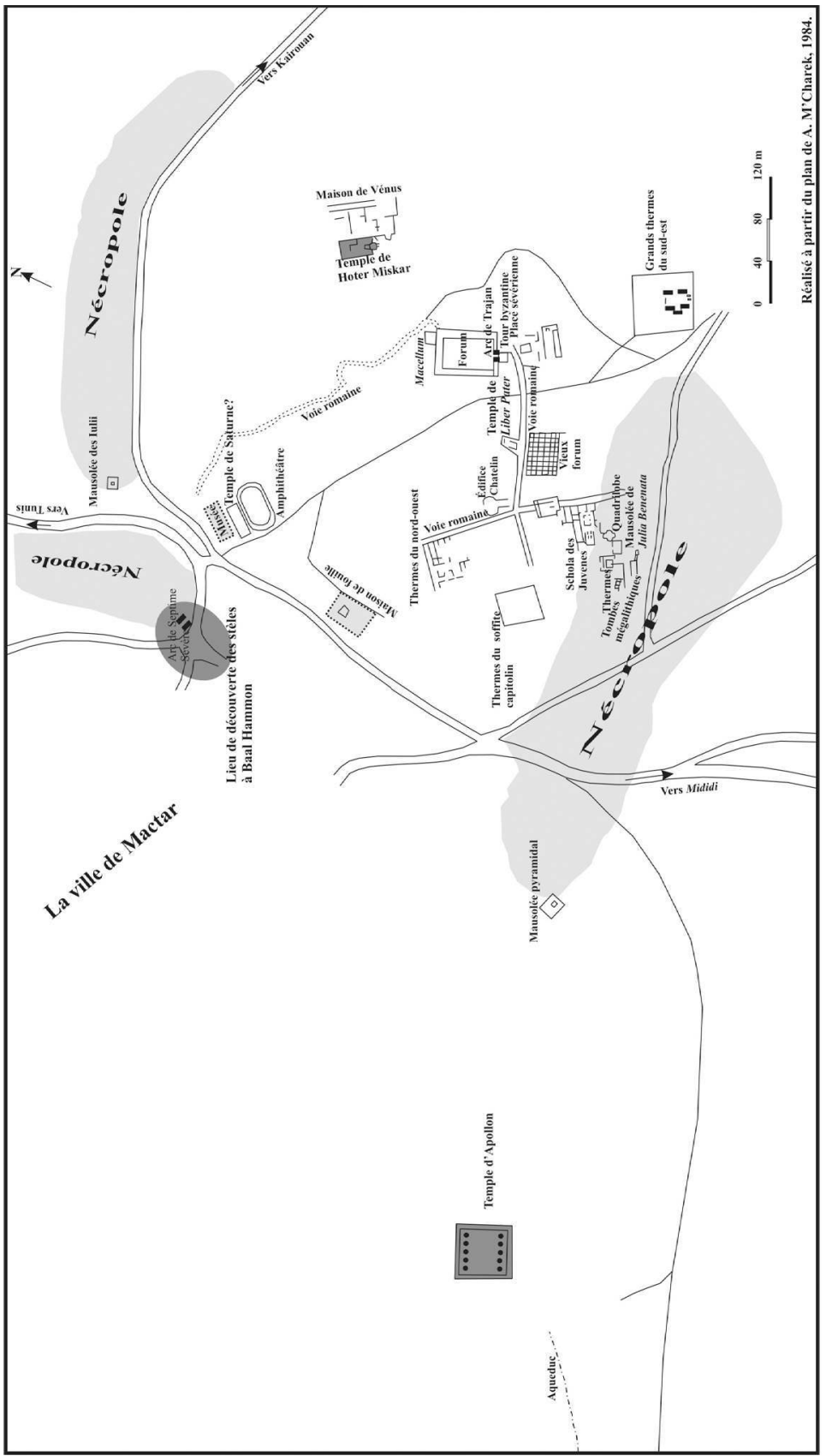

Fig. 2. Les lieux de culte préromains à Mactaris 